\title{
Retrieval of a dislodged and dismounted coronary stent; using a rendezvous and snare technique at the brachial artery level via femoral approach
}

\author{
Min-Woong Jeong ${ }^{1}$, Chang-Bae Sohn², Su Hong Kim², Jong-lk Park, Se-Ryeong Park ${ }^{1}$, Jun-Sik Min ${ }^{1}$ \\ ${ }^{I}$ Department of Internal Medicine, ${ }^{2}$ Cardiovascular Center, Busan Veterans Hospital, Busan, Korea
}

Coronary stent dislodgement during percutaneous coronary intervention, which occurs when the stent is passed through tortuous and calcified lesions, is not a rare complication. Without proper treatment, such as fixing with another stent in the coronary artery or removing the undeployed stent from the coronary artery or systemic artery system, this complication can cause serious problems. We experienced the unusual situation of a dismounted and dislodged coronary stent, in which retrograde retrieval to the radial artery was impossible during transradial coronary intervention. We report on use of a rendezvous and snare technique at the brachial artery level via femoral puncture, which resulted in resolution without surgery.

Keywords: Coronary; Stent; Endovascular procedures

\section{INTRODUCTION}

Stent dislodgement is a complication that can occur during percutaneous coronary intervention (PCI). In rare cases, without proper treatment, it can cause serious embolism complications. Various treatment methods have been introduced, most of which involve retrograde pull back of a stent. We encountered a dislodged and dismounted stent during transradial coronary intervention. Due to size discrepancy, performance of retrograde retrieval towards the transradial sheath was impossible. Therefore, antegrade retrieval was performed successfully through the femoral artery, and we reported this case.

Received: April 20, 2015, Revised: June 25, 2015

Accepted: July 01, 2015

Corresponding Author: Chang-Bae Sohn, Cardiovascular Medicine, Department of Internal Medicine, Busan Veterans Hospital, 420, Baegyang-daero, Sasang-gu, Busan 46996, Korea

Tel: +82-51-601-6386, Fax: +82-51-601-6727

E-mail: scb77@hanmail.net

\section{CASE}

A 65-year-old male patient was admitted to our emergency department due to mild continuous resting chest pain. He was on medication for hypertension and diabetes. Creatine kinase- $\mathrm{MB}$ and troponin-T levels remained at $17 \mathrm{U} / \mathrm{L}$ (range, $0-24 \mathrm{U} / \mathrm{L}$ ) and $0.17 \mu \mathrm{g} / \mathrm{L}$ (range, $0-0.1 \mu \mathrm{g} / \mathrm{L}$ ), respectively. Lowdensity lipoprotein level was $89 \mathrm{mg} / \mathrm{dL}$ (range, $0-140 \mathrm{mg} / \mathrm{dL}$ ). Electrocardiogram did not show ST segments elevation in any leads (Fig. 1). The transthorasic echocardiogram showed an ejection fraction of $60 \%$ and hypokinetic motion of the ante-

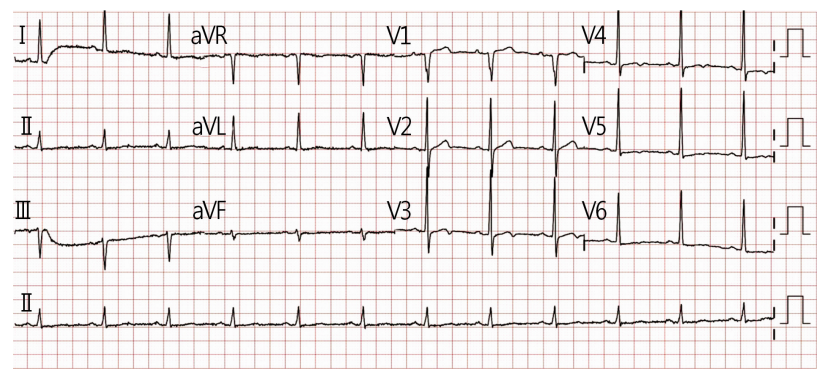

Fig. 1. Initial electrocardiogram does not show the elevation of the ST segment at precordial leads.

Copyright (c) 2016 Yeungnam University College of Medicine

This is an Open Access article distributed under the terms of the Creative Commons Attribution Non-Commercial License (http://creativecommons.org/licenses/by-nc/4.0/) which permits unrestricted non-commercial use, distribution, and reproduction in any medium, provided the original work is properly cited. 
roseptal regional wall of the left ventricle. Coronary angiography (CAG) showed over 90\% severe diffuse luminal narrowing on the proximal to middle left anterior descending artery (p-mLAD) and significant stenosis on the proximal left circumflex artery (pLCX). In addition, thrombus and aneurysmal changes were observed on the middle right coronary artery (mRCA) (Fig. 2). PCI on p-mLAD and pLCX lesions was planned and a right femoral artery puncture was performed. A 7Fr Judkins Left Guiding Catheter was engaged to the left main coronary artery and two coronary guidewires (Runthrough NS, Terumo Corporation, Tokyo, Japan) were positioned to his LAD and LCX, respectively. Intravascular ultrasound (IVUS) examination to evaluate the characteristics of plaque was attempted several times, but the IVUS catheter (VOLCANO, EagleEye Platinum, RX Digital catheter) could not pass through the LAD tight lesion due to insufficient flexibility of the tip. Because we had consumed too much time under non-ST elevation myocardial infarction state, we decided to perform angioplasty without IVUS examination. First, the anchor balloon technique was used in performance of predilatation using a $1.2 \times 15 \mathrm{~mm}, 1.5 \times 15 \mathrm{~mm}, 2.5 \times 15 \mathrm{~mm}$ balloon catheter on p-mLAD. Then, a $3.0 \times 30 \mathrm{~mm}$ and $2.5 \times 22 \mathrm{~mm}$ stent (Resolute Integrity, Medtronic, Medtronic Parkway, $\mathrm{MN}$, USA) was deployed on p-mLAD lesions. Next, predilatation was performed using a $2.0 \times 20 \mathrm{~mm}$ balloon catheter on the pLCX lesion and a $3.0 \times 22 \mathrm{~mm}$ (Resolute Integrity, Medtronic, Medtronic Parkway) stent was deployed. After the procedure, type $B$ dissection occurred on the distal edge of the mLAD stent. However, no chest pain or distal flow limitation was observ. Therefore we ended the procedure.

After the first PCI, dual anti-platelet therapy (aspirin and clopidogrel; $300 \mathrm{mg} / 600 \mathrm{mg}$ as loading dosage; $100 \mathrm{mg} / 75$ $\mathrm{mg}$, once a day as maintenance dosage) was administered for drug eluting stent and anti-coagulation therapy (enoxaparin sodium; $1 \mathrm{mg} / \mathrm{kg}$, twice a day as maintenance dosage; Clexane, Sanofi-Aventis, Maison Alfort, France) in order to reduce the size of RCA thrombus. A follow-up CAG check on the RCA thrombus was performed one week later. A 6Fr sheath was inserted through the right radial artery and decreasing thrombus size in the RCA lesion was observed. However the previous stent distal edge dissection and remnant luminal narrowing lesion were found on mLAD (Fig. 3). Therefore, adjunctive balloon angioplasty was performed using a $2.0 \times 15$ mm balloon catheter (Ikazuchi Rev, Kaneka Corporation,
Settsu, Osaka, Japan) and delivery of another stent was attempted using the buddy wire technique. However, during delivery of the other stent, the proximal part of the stent became dislodged as result of becoming stuck on the previous stent strut (Fig. 4A). First, the stent was carefully retrieved on the coronary artery and pulled back to the radial artery. However, retrieval to the sheath was impossible due to dislodgement (Fig. 4B). During several attempts to retrieve it into the guiding catheter, the stent became dismounted from the
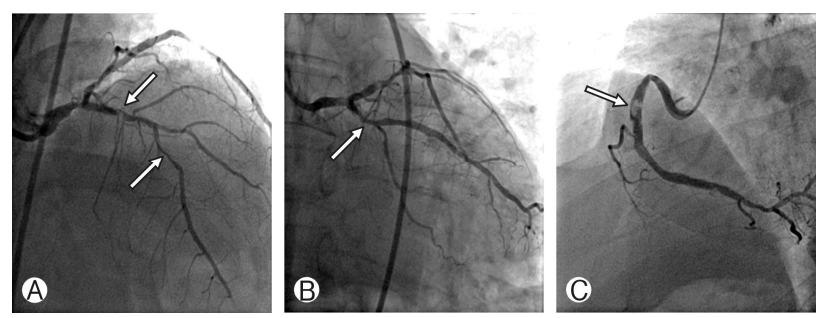

Fig. 2. Controlled coronary angiography. (A, B) There are multiple significant stenosis on the p-mLAD and Plcx (white arrow). (C) The thrombus is observed on the mRCA (white arrow). p-mLAD, proximal to the middle left anterior descending artery; pLCX, proximal left circumflex artery; mRCA, middle right coronary artery.
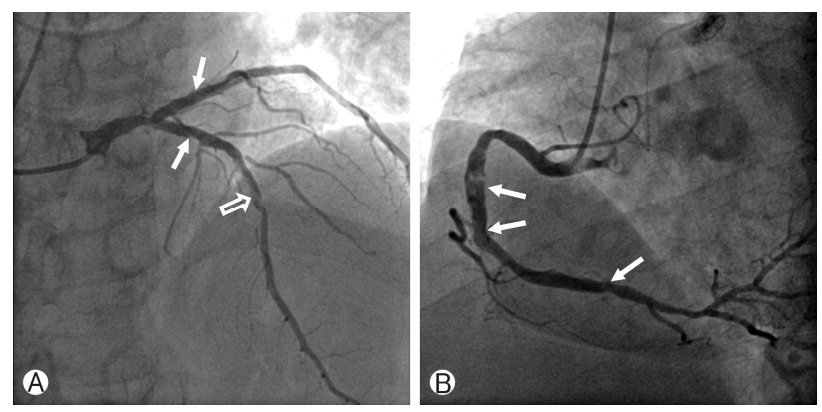

Fig. 3. Follow up coronary angiography. (A) Previous stent (white arrow) distal edge dissection (open arrow). (B) Coronary artery thrombus size decreased but still remained (white arrow).
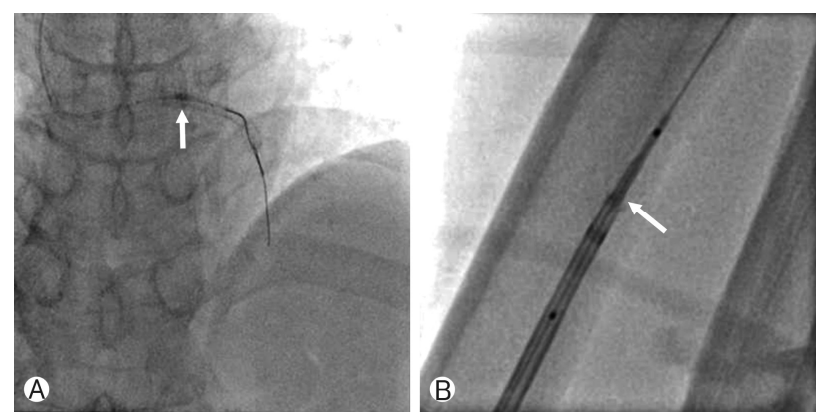

Fig. 4. (A) Occurrence of stent dislodgement (white arrow). (B) Dislodged stent could not retrieveinto $6 \mathrm{Fr}$ radial sheath (white arrow). 


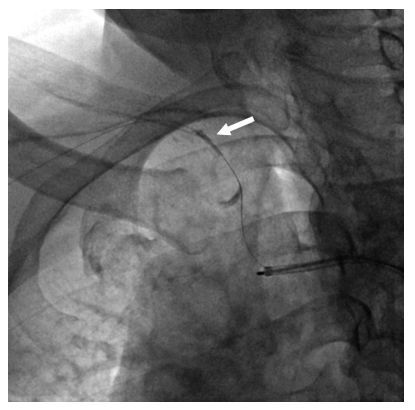

Fig. 5. Over the wire dismounted stent at the right subclavian artery level (white arrow).

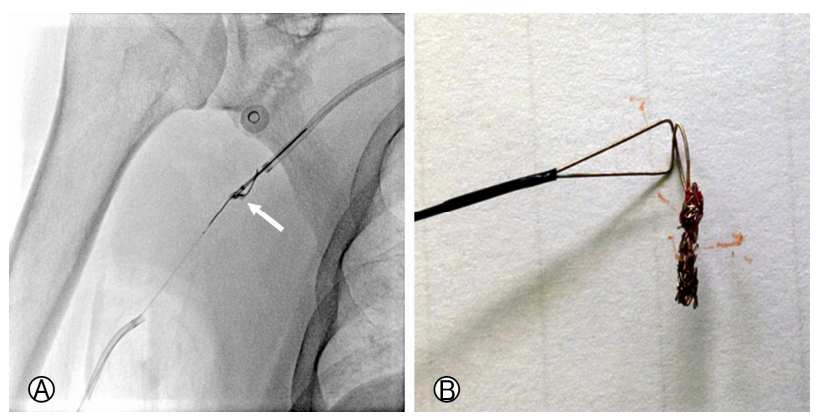

Fig. 6. (A) Stent retrieval via 7Fr sheath placed antegradely in the brachial artery. Over the wire dismounted stent was captured by snare (white arrow). (B) Successful exteriorization of the dislodged stent.

balloon catheter at the right subclavian artery level, so that the stent could no longer be manipulated (Fig. 5). The distal edge of the stent appeared to remain intact and the 7Fr MPA1 guiding catheter (Cordis Corporation, Miami Lakes, FL, USA) was introduced via the left femoral artery. The dismounted over-the wire stent was then pushed to the right brachial artery level to avoid migration of the dismounted over-the wire stent to the central circulation system. Antegrade retrieval of the dismounted and dislodged stent into the MPA1 catheter was attempted using a snare catheter system (MultiSnare, pfm medical mepro gmbh, Soterberg, Nonnweiler, Germany), which resulted in its successful removal from the patient's vascular system (Fig. 6). Fortunately, the CAG performed after the accident solution showed that mild flow limitation of mLAD remained, but the patient had no chest pain, therefore, we completed without the additional stent procedure and followed up with optimal medical therapy in order to prevent another complication. Afterwards, the patient continued outpatient treatment, continuing his work-out without chest pain. Follow-up CAG will be performed in the case of recurrence of effort-related chest pain.

\section{DISCUSSION}

Stent dislodgement, which can occur during coronary intervention, is not a rare complication. Without treatment, the coronary stent becomes a risk for myocardial infarction [1]. Stent dislodgement can occur under the following conditions: severe angulation and tortuosity; diffuse and calcified lesion; previous underexpanded stent. The reported incidence of dislodgement is approximately $0.32-8 \%$ [2]. When stent dislodgement occurs, removal from the arterial system is the best option and there are various retrieval techniques: catheter balloon inflated distal to the undeployed stent (small-balloon technique) [3], retrieval using a snare [4] or forceps [5], stent entrapment with two twisted guidewires [6], combination of rotablation and snaring [7], and basket devices [8]. When a retrieval method is not feasible, 'bail-out crush technique' using another stent is another option. Recently, transradial coronary intervention has been performed frequently and arterial sheath and guiding catheters of less than $6 \mathrm{Fr}$ are often used. Proper radial artery diameter should be secured in order to eliminate over-the-wire stent using the snare through the transradial approach introduced by Kiemeneij et al. [9]. If this is not possible, another option is to pull it back to the peripheral artery and perform an adjunctive vascular surgery. Another treatment option is antegrade removal using a larger forcep or snare catheter of over 7Fr with a transfemoral approach. However, when performing the procedure at the ascending aorta level, there is a risk of causing stent embolism to the cerebral artery. Thus, we believe that it is safer to advance a transfemoral catheter to the brachial or brachioradial junction. Nowadays, various vascular compression devices can be used to stop bleeding of the femoral artery. Thus, it is possible to reduce occurrence of post-procedure complications such as hematoma and pseudoaneurysm, which can occur when using the transfemoral approach. In our case, the proximal portion of the stent was dislodged while positioning an additional stent during subsequent PCI. Despite our attempts to retrieve it to the $6 \mathrm{Fr}$ transradial sheath, the size of the radial artery and sheath was too small to capture the dislodged stent. As a result of numerous retrieval attempts, manipulation was no longer possible as the stent had become dismounted from the balloon catheter (over-the-wire stent) at the subclavian artery level. However, the dislodged and dismounted 
stent was removed using a 7Fr guiding catheter and snare at the brachial artery level using the antegrade retrieval technique and transfemoral approach without surgery. The process and challenges are explained in this case report.

\section{REFERENCES}

1. Shiojima I, Ikari Y, Abe J, Ishizaka N, Maemura K, Kurihara $\mathrm{H}$, et al. Thrombotic occlusion of the coronary artery associated with accidental detachment of undeployed Palmaz-Schatz stent. Cathet Cardiovasc Diagn 1996;38:360-2.

2. Kwan TW, Chaudhry M, Huang Y, Liou M, Wong S, Zhou X, et al. Approaches for dislodged stent retrieval during transradial percutaneous coronary interventions. Catheter Cardiovasc Interv 2013;81:E245-9.

3. Rozenman Y, Burstein M, Hasin Y, Gotsman MS. Retrieval of occluding unexpanded Palmaz-Schatz stent from a saphenous aorto-coronary vein graft. Cathet Cardiovasc Diagn 1995;34: 159-61.
4. Elsner M, Peifer A, Kasper W. Intracoronary loss of balloonmounted stents: successful retrieval with a 2 mm-"Microsnare"device. Cathet Cardiovasc Diagn 1996;39:271-6.

5. Kim MH, Cha KS, Kim JS. Retrieval of dislodged and disfigured transradially delivered coronary stent: report on a case using forcep and antegrade brachial sheath insertion. Catheter Cardiovasc Interv 2001;52:489-91.

6. Antonellis IP, Patsilinakos SP, Pamboukas CA, Kranidis AJ, Papadopoulos A, Margaris N, et al. Successful withdrawal from the right coronary artery of an NIR stent dislodged from the balloon catheter. J Interv Cardiol 1999;12:215-7.

7. Kim JH, Jang WJ, Ahn KJ, Song YB, Hahn JY, Choi JH, et al. Successful retrieval of intravascular stent remnants with a combination of rotational atherectomy and a gooseneck snare. Korean Circ J 2012;42:492-6.

8. Eggebrecht H, Haude M, von Birgelen C, Oldenburg O, Baumgart D, Herrmann J, et al. Nonsurgical retrieval of embolized coronary stents. Catheter Cardiovasc Interv 2000;51:432-40.

9. Kiemeneij F, Laarman G. Transradial coronary angioplasty. CD-ROM. Physicians' Multimedia Publishers; 1999. 